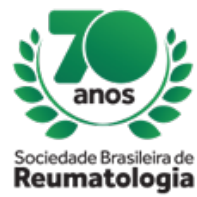

\title{
ANCA POSITIVE VASCULITIS CAUSING PULMONARY-RENAL SYNDROME'S IN A PATIENT WITH RHEUMATOID ARTHRITIS
}

lane Tamara Dondé (Hospital Regional de Mato Grosso do sul, Campo Grande, MS, Brasil), Eduardo Ráfaga Gonçalves Calvoso (Hospital Regional de Mato Grosso do Sul, Campo Grande, MS, Brasil), Hellen Tavares Franco Calvoso (Universidade Anhanguera Uniderp, Campo Grande, MS, Brasil), Renata Vaz de Oliveira (Hospital Regional de Mato Grosso do Sul, Campo Grande, MS, Brasil), Querolai Gomes Gadelha

(Hospital Universitário Maria Aparecida Pedrossian- Universidade Federal de Mato Grosso do Sul, Campo Grande, MS, Brasil), Alex Magno Coelho Horimoto (Hospital Regional de Mato Grosso do Sul, Campo Grande, MS, Brasil), Luiz Armando Pereira Patusco (Hospital Regional de Mato Grosso do Sul, Campo Grande, MS, Brasil), Liana Peres Duailibe (Hospital Regional de Mato Grosso do Sul, Campo Grande, MS, Brasil), Andressa Mateus da Cunha (Hospital Regional de Mato Grosso do Sul, Campo Grande, MS, Brasil)

\section{BACKGROUND}

The presence of ANCA antibodies in rheumatoid arthritis is frequent, but they aren't associated with the RA activity, cutaneous vasculitis or glomerular disease. In regard to ANCA associated vasculitis (AAV) and RA overlap syndrome, there are about 35 case reports in literature. In almost all of them, the AAV is microscopic polyangiitis (MP). In those cases, practically all course with kidney manifestations. Illegal drugs like cocaine may also trigger immune-mediated vasculitis, as well as other medications like sirolimus, D penicillamine, propylthiouracil, and diphenylhydantoin. One study report a case of AAV in RA after the use of infliximab.

\section{CASE REPORT}

Female, 45 years old, RA diagnosed since 2005 (RF and anti- CCP positive), in regular use of methotrexate, prednisone, and adalimumab; Comorbidities: arterial hypertension, diabetes, smoking and former use of marijuana. Admitted in the hospital with progressive dyspnea and hemoptysis a week ago, developed with respiratory failure and need for ventilatory support. The patient also presented acute renal failure and need for intermittent hemodialysis. Laboratory tests showed a hemoglobin of $4,1 \mathrm{mg} / \mathrm{dL}$, leukocytosis of 13.84; normal account of platelets; $C R P=7,5 \mathrm{mg} / \mathrm{dL} ; B U N=43.4$ and creatinine $=3,0 \mathrm{mg} / \mathrm{dl}$. Initial chest $X$-ray and $C T$ revealed interstitial, diffuse and bilateral infiltrates, suggestive of alveolar bleeding. Serology for hepatitis and HIV were negative. The ratio protein/creatinine in urine was 2,88g. Renal biopsy present segmental and focal sclerosing glomerulonephritis, with diffuse crescents; a pattern of rapidly progressive glomerulonephritis; immunofluorescence showed discrete glomerular deposits of IgG and IgM; a pauciimmune lesion pattern. P-ANCA was $1 / 80$ positive, and C-ANCA negative.

She underwent pulse therapy with methylprednisolone on two occasions due to recurrence of alveolar hemorrhage. Evolved with nosocomial complications, successfully treated. After that, was submitted to pulse therapy with cyclophosphamide. She was discharged from hospital to follow-up after improvement of pulmonary images and renal function.

\section{CONCLUSION}

The AAV is rarely related to other autoimmune diseases, but there are few reported cases of RA and AAV. The recognition of this overlap syndrome is important for sorological screening and early recognition. Possible reasons for the association between ANCA systemic vasculitis and rheumatoid arthritis may be the common genetic predispositions to autoimmunity which involve HLA region or genes, besides the use of TNF antagonists which may have predisposed to the development of the secondary autoimmune disease. 